Detailed Description of Electronic Microscope

\title{
Stage Manipulator
}

\author{
E. J. Rogers
}

Figure 1 shows the scanning pattern through which the slide is moved. The three sketches illustrate the adjustment of the motion limits to suit the desired area and the adjustment of the vertical step size to prevent underscanning or overlap when the aperture size is changed. The horizontal and vertical limits of motion, horizontal rate and vertical step size are preset by the operator. Once initiated, the scanning process proceeds automatically to completion without further attention. The completion of each horizontal sweep is registered by the Sodeco counter on the control panel.

The scanning parameters are independently adjustable over the following ranges:

$\begin{array}{ll}\text { vertical range } & 5 \text { to } 100 \mu \\ \text { horizontal range } & 5 \text { to } 100 \mu \\ \text { horizontal rate } & 0.4 \text { to } 4 \mu / \mathrm{sec} \\ \text { vertical step size } & 0.2 \text { to } 10 \mu\end{array}$

\section{LEGAL NOTICE}

This report was prepared as an account of Government sponsored work. Nelther the United States, nor the Commission, nor any person acting on behalf of the Commission:
A. Makes any warranty or A. Makes any warranty or representation, expressed or implied, with respect to the accu-
-acy, completeness, or usefulness of the information contained in this report, $\mathrm{I}$ any information, apparatus, method, or process disclased in this report, or that the use rivately owned rights; or

B. Assumes any liabillties with respect to the use of, or for damages resulting from the
of any information, apparatus, method, or process disclosed in to As used in the above, "person acting on behalf of the Cod in this report.

pioyee or contractor of the Commission, or employee of such contractor, foludes any emsuch employee or contractor of the Commission, or employee of such contrator extent that with the Cos or provides access to, any information pursuant to his employment or contract
Facsimile Price \$ Microfilm Price $\$$

Available from the Office of Technical Services Department of Commerce Washington 25, D. C. 


\section{DISCLAIMER}

This report was prepared as an account of work sponsored by an agency of the United States Government. Neither the United States Government nor any agency Thereof, nor any of their employees, makes any warranty, express or implied, or assumes any legal liability or responsibility for the accuracy, completeness, or usefulness of any information, apparatus, product, or process disclosed, or represents that its use would not infringe privately owned rights. Reference herein to any specific commercial product, process, or service by trade name, trademark, manufacturer, or otherwise does not necessarily constitute or imply its endorsement, recommendation, or favoring by the United States Government or any agency thereof. The views and opinions of authors expressed herein do not necessarily state or reflect those of the United States Government or any agency thereof. 


\section{DISCLAIMER}

Portions of this document may be illegible in electronic image products. Images are produced from the best available original document. 


\section{Description of Stage}

The mechanical principle of the stage is illustrated diagramatically in the sketch, Fig. 2. Two control assemblies are used. One assembly is supported by and rotated $90^{\circ}$ with respect to the other so that the horizontal and vertical positions can be controlled independentiy.

The 0.005 in. tungsten wires used for both horizontal and vertical control are $9.2 \mathrm{~cm}$ long. For the full 100 micron range the wire temperature swings from $100^{\circ} \mathrm{C}$ to $350^{\circ} \mathrm{C}$. Over this temperature range the resistance of the wire changes from 0.5 ohm to $0.95 \mathrm{ohm}$. The relation between the wire resistance and mechanical displacement is, for all practical purposes, linear. Under average ambient conditions, the feedback-controlled dc heating current in the wire varies from about 0.5 to 1.2 amps for full displacement. The control wires are run through 5/16 in. glass tubes for mechanical protection and shielding from drafts. The entire mechanical stage assembly is shown in the photograph, Fig. 3 .

Functional Description of Electronic Control System

As seen in Fig. 4, the electronic control system is divided into four basic functional parts. The horizontal and vertical wire control circuits, which are identical, produce a direct heating current in each control wire. The heating current is feedback-controlled to adjust the temperature of the wire to cause its resistance to be accurately proportional to the voltage 
applied to the wire control input. Because both the resistance and the thermal expansion of the wire are invariantly related. to its temperature, the position of the stage is determined by the controlling voltage.

Tungsten was chosen for the control wire because its high ratio of thermal coefficient of resistivity to thermal coefficient of expansion yields a comparatively large resistance charge for a given stage displacement.

The horizontal programmer produces a triangular output voltage of magnitude and slope adjusted to move the stage through the desired distance at the desired rate. At the end of each. horizontal sweep the horizontal programmer delivers a trigger pulse to the vertical programmer, causing it to add an increment to the vertical position control voltage, thus advancing the stage one step vertically. This process is repeated until the vertical control voltage reaches a preset value which represents the height of the rectangular area to be scanned. At this point the vertical and horizontal programmers are reset and the stage is returned to the starting position. An electromechanical counter on the vertical programmer panel registers each horizontal sweep and is not reset until the next scanning cycle is initiated. Wire Control Circuits

Figure 5 shows a schematic diagram of the identical horizontal and vertical wire control circuits. The control wire Is heated by dc which flows in the collector circuit of the germanium power transistor, $Q_{7}$. A $67 . \mathrm{ma}, 10 \mathrm{kc}$, square wave 
sensing current signal is applied simultaneously to the control wire by $Q_{5}$ to provide an ac voltage drop in the wire which represents the resistance and, therefore, the temperature of the wire.

A $10 \mathrm{kc}$ current of the same phase is applied to the $1.5 \mathrm{ohm}$ resistor, $R_{1}$, by $Q_{6}$. The magnitude of this current is proportional to the collector supply voltage of $Q_{6}$ which is the -8 to $15 \mathrm{~V}$ wire control voltage. For any particular control voltage there exists a wire temperature at which the $10 \mathrm{kc}$ voltages across the wire and $R_{1}$ are equal. The phase and. magnitude of the voltage difference between the wire and $R_{1}$ therefore represent the sign and magnitude of the wire temperature error.

The ac error signal is amplified by the following two-stage push-pull amplifier and applied to the synchronous rectifier, $Q_{12}$ and $Q_{13}$. The resulting dc signal is amplified by $Q_{14}$ to drive the base of $Q_{7}$ to provide the required heating current. When the stage position is run through the $100 \mu$ range, the peak to peak value of the $10 \mathrm{kc}$ voltage across the wire increases by $100 \mathrm{mv}$. The corresponding increase in error signal, measured between the wire and $R_{1}$, is approximately $1 \mathrm{mv}$, indicating a $100 \mathrm{p}$ gain of the order of 100 which is more than adequate to insure that the stage position is essentially independent of the amblent temperature.

The $1.5 \mathrm{ohm}$ reference resistor, $R_{1}$, is located at the stage. Its ground return is common with that of the control wire, so 
that the voltage drop in the common ground return which results from the difference between the $10 \mathrm{kc}$ currents in the wire and in $R_{l}$ appears to the push-pull amplifier input as a common mode signal and is rejected. Separate potential and current leads are run out to the control wire and to $R_{1}$ to prevent the current lead voltage drops from appearing at the amplifier input.

Feedback due to the large collector-to-base capacitance of $Q_{7}$ was found to cause the impedance in its collector circuit to be so low at $10 \mathrm{kc}$ as to cause serious shunting of the wire signal. This effect was eliminated by the addition of emitter degeneration via the $360 \mu h$ ferrite-cored inductor, $L_{1}$, to yield a high output 1mpedance at $10 \mathrm{kc}$.

\section{Dynamic Stability}

Ideally, signals would propagate around the loop only in the normal mode in which they appear as modulation on the $10 \mathrm{kc}$ carrier produced by changes in the wire resistance. Due to unbalance in the synchronous rectifier and other circuit imperfections it is also possible for signals to propagate around the loop by direct amplifioation. Since the tungsten wire is a very Insensitive modulator a loop gain of 100 in the desired mode requires that the total electronic amplification in the loop be of the order of several thousand times. The resulting gain in the direct mode, therefore, can be of the order of hundreds.

In the modulated carrier mode the long thermal time constant of the wire sets a maximum frequency response which is at least 
3 orders of magnitude below. the electrical bandwidth of the system. The response extends down to dc at the low end. These conditions are 1deal from the stability standpoint: The stability situation is quite different for direct mode signals. Stability at the high end is no problem because the rectifier filter capacitor, $\mathrm{C}_{3}$, sets the upper cutoff frequency below the point where other circuit elements can contribute the additional $90^{\circ}$ phase lag required for oscillation. At the low end, however, phase leads are contributed by the interstage coupling capacitors, the low frequency characteristic of transformer, $\mathrm{T}_{2}$, and the output capacitor of the $5 \mathrm{~V}$ power supply. Here we have numerous time constants in the same order of magnitude, a very unfavorable situation for stability. The system was indeed observed to oscillate at about $200 \mathrm{cps}$.

It was not found feasible to separate the values of the pertinent time constants widely enough to eliminate the oscillation. The instability was finally eliminated by the addition of the lowpass filter formed by $\mathrm{R}_{2}$ and $\mathrm{C}_{5}$ at the synchronous rectifier output. The resulting upper cutoff frequency is much lower than the lower cutof frequency for the direct amplification mode. The gain in this mode is therefore drastically reduced at all frequencies and no oscillation occurs.

The aforementioned filter also affects the upper frequency cutoff in the modulated carrier mode. Its time constant of about $0.03 \mathrm{sec}$ (including loading by $Q_{14}$ ) is sufficiently shorter 
than the thermal time constant of the wire that the stability in this mode is not affected. If $\mathrm{C}_{5}$ is increased to the order of $200 \mu \mathrm{f}$ the preceding statement no longer holds and the system w1ll overshoot seriously when the control voltage is changed quickly.

\section{Horizontal Programmer}

A schematic of the horizontal programmer appears in Fig. 6 . The triangular horizontal control voltage wave is generated by a Miller integrator utilizing a Phllbrick Type P-2 operational amplifier. Its gain of over 40,000 and its input current of less than $5 \times 10^{-11}$ amp provide excellent linearity and stability even at the lowest scanning rates.

The feedback amplifier of unity voltage gain, comprised of $Q_{9}, Q_{10}, Q_{11}$ and $Q_{12}$, delivers an output voltage equal to the integrator output voltage at the 25 to $50 \mathrm{ma}$ current level required to drive the horizontal wire control circuit. A 100p gain of several hundred times insures that the output voltage faithfully follows that of the integrator at an output impedance of less than 1 ohm.

In the quiescent condition the integrator capacitor is shorted by $R_{y 3}$ and the output voltage is held equal to the $-8 \mathrm{~V}$ reference voltage applied to input terminal 1 of the $\mathrm{P}-2$ amplifier. This voltage, which may be set by adjusting $R_{1}$, determines the standing current in the horizontal control wire. The integrator output voltage will rise or fall according to the state of the flip-flop, $Q_{2}$ and $Q_{3}$, at a rate determined by the setting of $R_{i 2}$, the horizontal rate control. 
The output voltage is sensed by two difference amplifiers, $Q_{13,14}$ and $Q_{15,16}$, used as amplitude discriminators to set the upper and lower limits of the horizontal sweep. The negative reference voltage for the lower limit discriminator, applied to the base of $Q_{16}$, is held 0.2 volt more negative than the integrator reference voltage by the forward drop in the germanium diode, $D_{1}$. In the quiescent condition the output voltage is therefore 0.2 volt less than the discriminator threshold and the discriminator output current holds $Q_{6}$ in saturation. The pulse gate comprised of $Q_{6}$ and $Q_{4}$ applies positive trigger pulses, derived from the $10 \mathrm{kc}$ square wave output of the horizontal wire control unit, to the collector of $Q_{3}$, setting the flip-flop in the proper state to produce a voltage near zero at the collector of $Q_{5}$, resulting in a positive integrator input voltage.

When the "start" button on the vertical programmer unit is pressed, $\mathrm{R}_{\mathrm{y} 3}$ opens, unshorting the integrator capacitor. The negative integrator output voltage increases at a uniform rate. When the output voltage, wh1ch is applied to the base of Q $13^{\text {" }}$ exceeds the dc voltage on the base of $Q_{14}$, set by the horizontal range control, $R_{3}$, in the base circuit of $Q_{17}$, the upper limit discriminator, $Q_{13,14}$, feeds current to the base of $Q_{18}$, saturating it. Positive trigger pulses are now applied to the collector of $Q_{2}$, reversing the flip-flop to produce a voltage. near -25 at the emitter of $Q_{5}$ and hence a negative integrator 
input voltage. The negative integrator output voltage decreases at a uniform rate until it crosses the lower limit discriminator threshold. This discriminator and its pulse gate then reverse the flip-flop and the output voltage starts upward again. The horizontal programmer voltage thus oscillates linearly between the two preset limits as long as the integrator capacitor remains unshorted, producing a uniform back and forth horizontal motion of the stage. At each transition of the flip-flop the vertical trigger generator, $Q_{19}, Q_{20}$ and $Q_{21}$, delivers a positive trigger pulse to the vertical programmer to advance the vertical position by one step. When the desired vertical limit is reached the integrator capacitor is shorted and the horizontal programmer is returned to the quiescent position.

Vertical Programmer

The vertical programmer is shown schematically in Fig. 7 . When the "start" button is pressed the Sodeco counter "reset" coil is energized, clearing the counter, and the "latch" coll of latching relay, $R_{Y I}$, is energized placing contacts $1 \mathrm{~A}$ in the indicated position. This operation applies $-25 \mathrm{~V}$ to the upper end of the "release" coil, 1lluminates the "scan" light on the front panel, energizes relay, $\mathrm{R}_{\mathrm{y} 3}$, in the horizontal programmer, removes the $-25 \mathrm{~V}$ inhibiting bias from the gating diode, $D_{1}$, and de-energizes the 4 pdt relay, $R_{y 2}$, placing its contacts in the position shown. 
$R_{y 3}$ unshorts the integrator capacitor in the horizontal programmer and the horizontal sweeping process commences. At the end of each horizontal sweep, the vertical trigger pulse generated by the horizontal programmer is passed by the gating diode, $D_{1}$, to trigger the univibrator, $Q_{1}$ and $Q_{2}$, which produces a $50 \mathrm{msec}$ output pulse. This pulse is amplified to the necessary 2 amperes by $Q_{3}, Q_{4}$ and $Q_{5}$, and delivered to the Sodeco counter "count" coil and to the combination of stepping switches, SSI and SS2, to advance their position by one step, increasing the resistance between points $X$ and $Y$ (located between $Q_{16}$ and $Q_{17}$ ) by $100 \mathrm{ohms}$. This resistance increment, by a process involving $\dot{Q}_{12}$ through $Q_{22}$, to be described later, increases the vertical programmer negative output voltage by an increment whose value is adjusted by the vertical step size control, $R_{1}$, and the stage position is advanced one step vertically.

The output voltage is compared by the difference amplifier, $Q_{9}$ and $Q_{10}$, with a preset voltage selected by the vertical range control, $R_{2}$ (located between $Q_{11}$ and $Q_{12}$ ). When the output voltage corresponding to the desired vertical range is reached, the dc output of the difference amplifier triggers the Schmitt discriminator, $Q_{7}$ and $Q_{8}$, whose output current saturates $Q_{16}$ to energize the release coil of $R_{y l}$, unlatching that relay and energizing $R_{y 2}$. Relay, $R_{y_{3}}$, shorts the integrator capacitor in the horizontal programmer, returning it to the quiescent state. Contacts $2 A$ and $2 B$ switch the stepping switches to the 
self-homing mode while $2 \mathrm{C}$ shorts the stepping switch resistor string, returning the output voltage immediately to the quiescent value. $R_{y l}$ may be released to initiate the "turn-off" sequence at any time before the vertical limit is reached by pressing the "stop" button (near $Q_{6}$ ) to energize the release coll. If the vertical step size is adjusted so that the limit of 103 steps provided by the stepping switches is reached before the selected vertical range can be scanned, the turn-off sequence is initiated by the " $A$ " circuit of $R_{y 2}$ on the last step.

In all cases the counter is not reset until the beginning of the next scanning process so that the total number of horizontal sweeps is displayed until the next scanning cycle is initiated.

Considerable difficulty has been experienced with the build-up of an insulating film on the contacts of stepping sw1tches (and other types of relays) when the contacts were operated at low voltages and currents. The use of special contact materials greatly alleviates the problem but does not eliminate It altogether. For this reason the use of the realotor otring as a simple unloaded voltage divider in which the position of the output tap is selected by the stepping switches is unsatisfactory. To draw current through the contacts would require loading the output, producing a non-linear advance in output voltage unless the divider resistors were weighted accordingly and errors might still result from residual contact resistance. 
The use of the amplifier comprised of $Q_{12}$ through $Q_{16}$ avoids the contact-resistance problem completely. A feedbackcontrolled, constant output current from the collector clrcuit of $\mathrm{Q}_{19}$ flows through the stepping switch contacts, through whatever portion of the resistor string is in the circult and through $R_{1}$ and $R_{3}$ to ground. The base, $Q_{13}$, which is one side of the differential input of the amplifier is connected to an adjustable reference of voltage, $V_{R}$, of approximately -8 volts, while the voltage drop across $R_{1}$ and $R_{3}$ due to the output current is applied to the base of $Q_{16}$, the other input terminal. The amplifier gain of several thousand times insures that the drop across $R_{2}$ and $R_{3}$ will be very nearly equal to $v_{R}$ and therefore that the output current,

$$
i_{0}=\frac{V_{R}}{R_{1}+R_{3}} \text {, where } v_{R} \text { is the voltage }
$$

applied to the base of $\mathrm{Q}_{13}$.

The voltage at the top of the nth $100 \mathrm{ohm}$ resistor, and all points above it, when the stepping switch combination is in the nth position will be given by $v_{n}=v_{R}+100 \mathrm{ni}_{0} \cdot V_{n}$ is independent of any contact voltage drop in the switch provided only that the drop is not so large as to prevent proper functioning of the circuit.

$R_{4}$, which sets $V_{R}$, establishes the voltage at the bottom of the string while $R_{1}$ sets $i_{0}$ and, therefore, the voltage increment for each step. The output voltage is taken from the 
top of the resistor string and delivered to the vertical wire control at the necessary high current and low output impedance by the output amplifier, $Q_{17}$ through $Q_{22}$. This amplifier is very similar to its counterpart on the horizontal progranmer except that by the use of silicon transistors, operating at high impedance levels, its input impedance is made higher than one megohm. The high input impedance is necessary to prevent error due to voltage drop in the portion of the resistor string which is above the stepping switch tap position.

In a manner analogous to that of the horizontal programmer, the voltages which determine the quiescent stage output voltage, the vertical step size and the vertical range are all derived from and proportional to the -2OV supply voltage which supplies the $10 \mathrm{kc}$ reference current to the vertical control wire. The stage position is therefore essentially unaffected by supply voltage variations. 


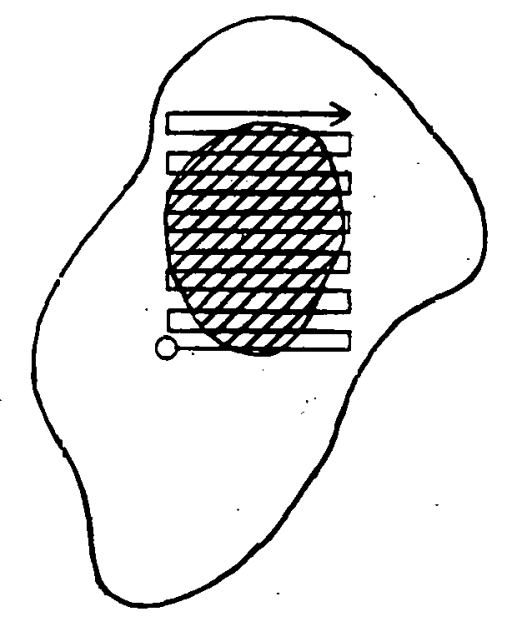

$\underline{A}$

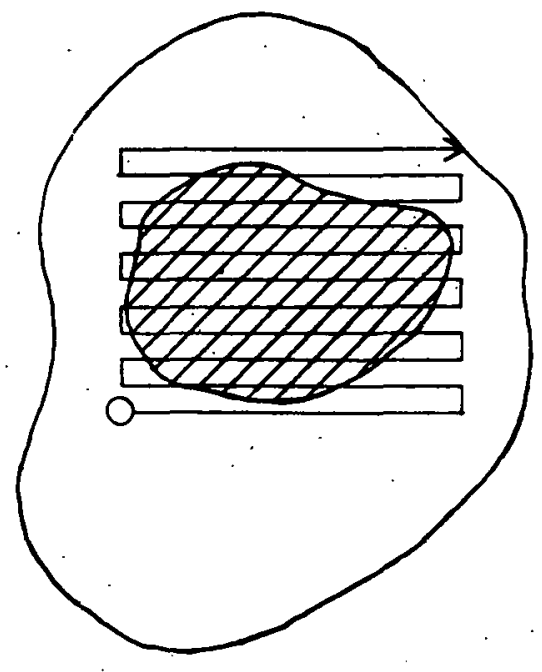

$\underline{B}$

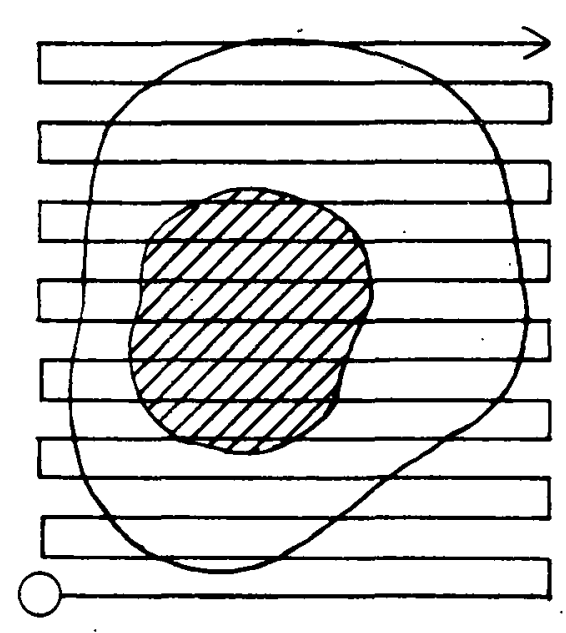

$\underline{C}$

Fig. 1 Scanning pattern - The sketches $A, B$ and $C$ show how the vertical and horlzontal ranges are adjusted to frame the desired area. Different apertures are shown with their corresponding vertical step sizes to illustrate the adjustment of step size to prevent overlap or underscanning when the aperture is changed. 


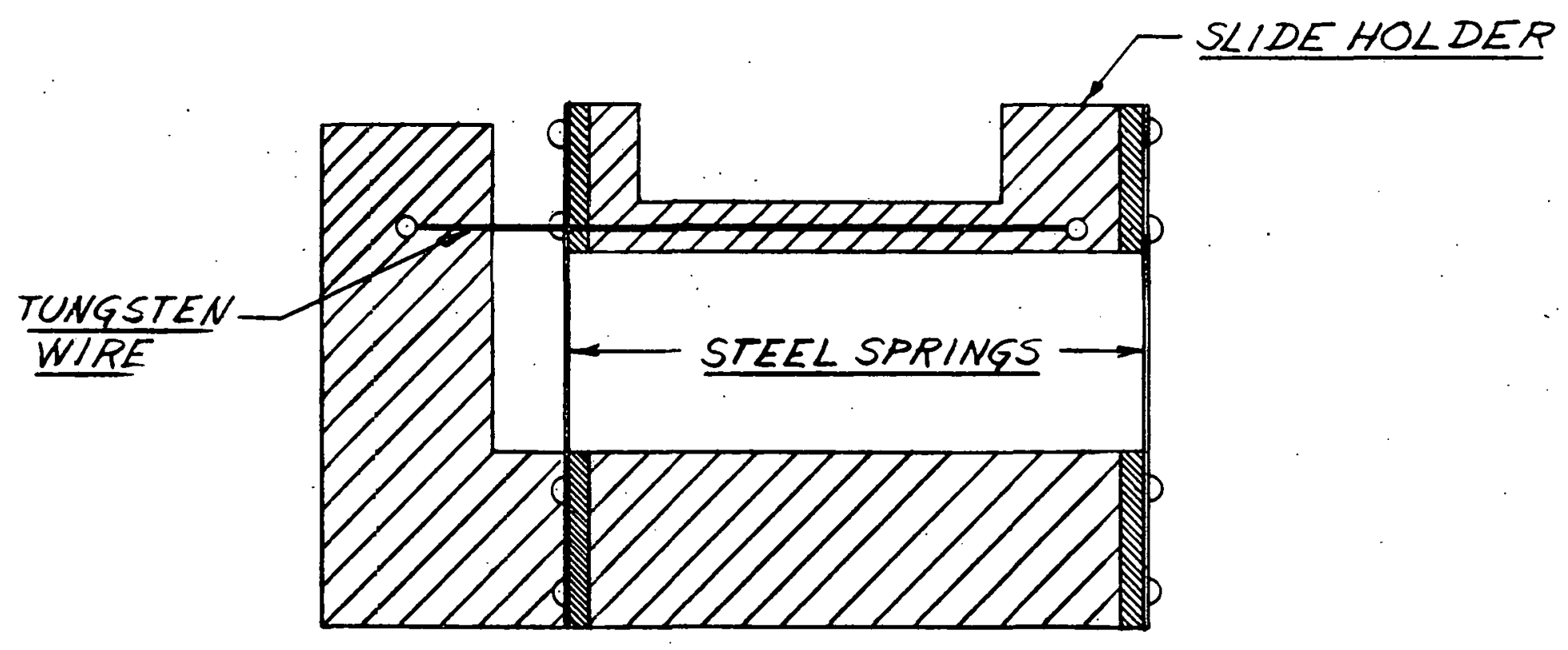

Fig. 2 Sketch 1llustrating the mechanical principle of the stage. The tungsten wire is held under tension by the steel leaf springs. Thermal expansion and contraction of the tungsten wis produce the stage motion. 


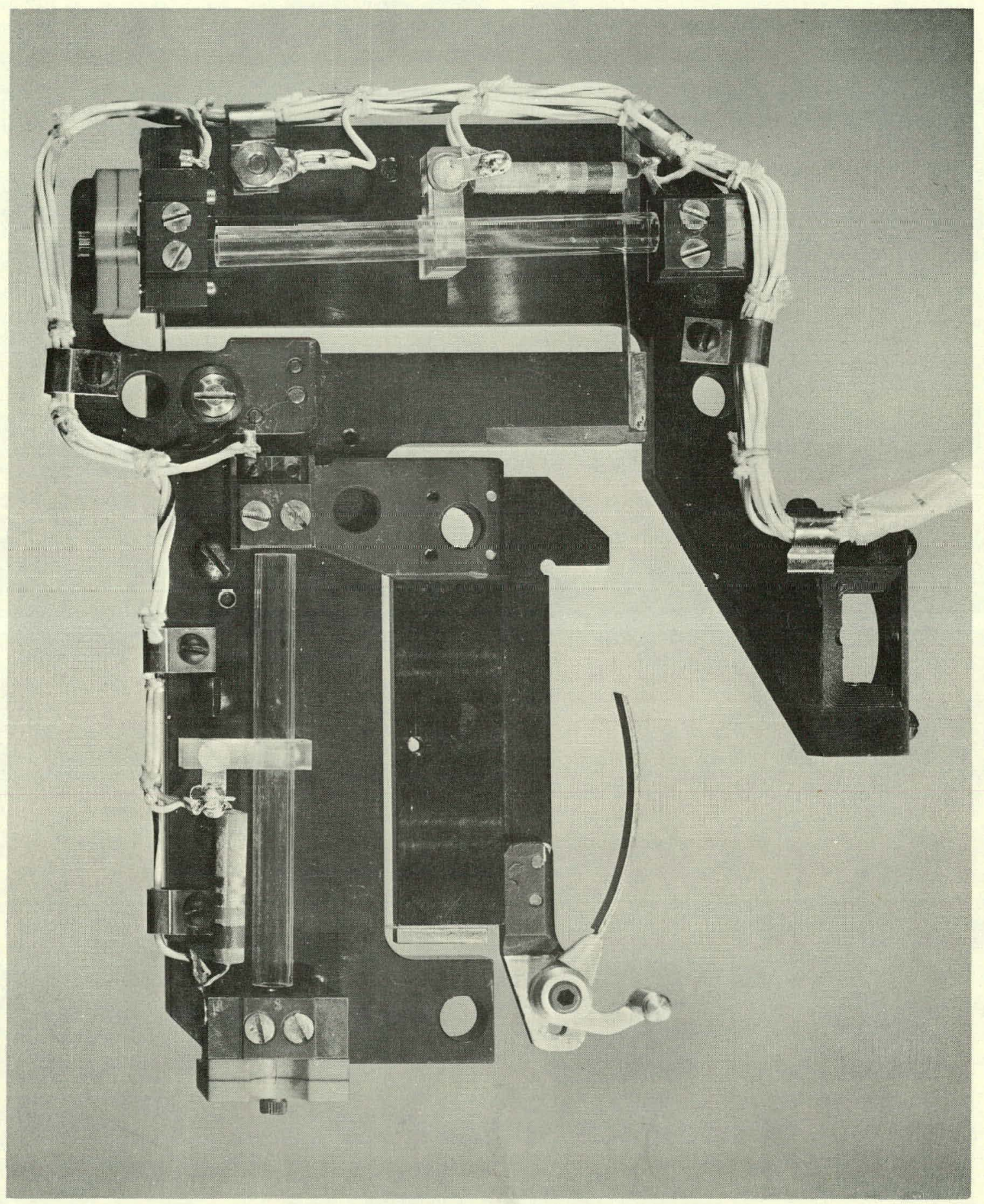

Fig. 3 Photograph of the complete stage assembly. 


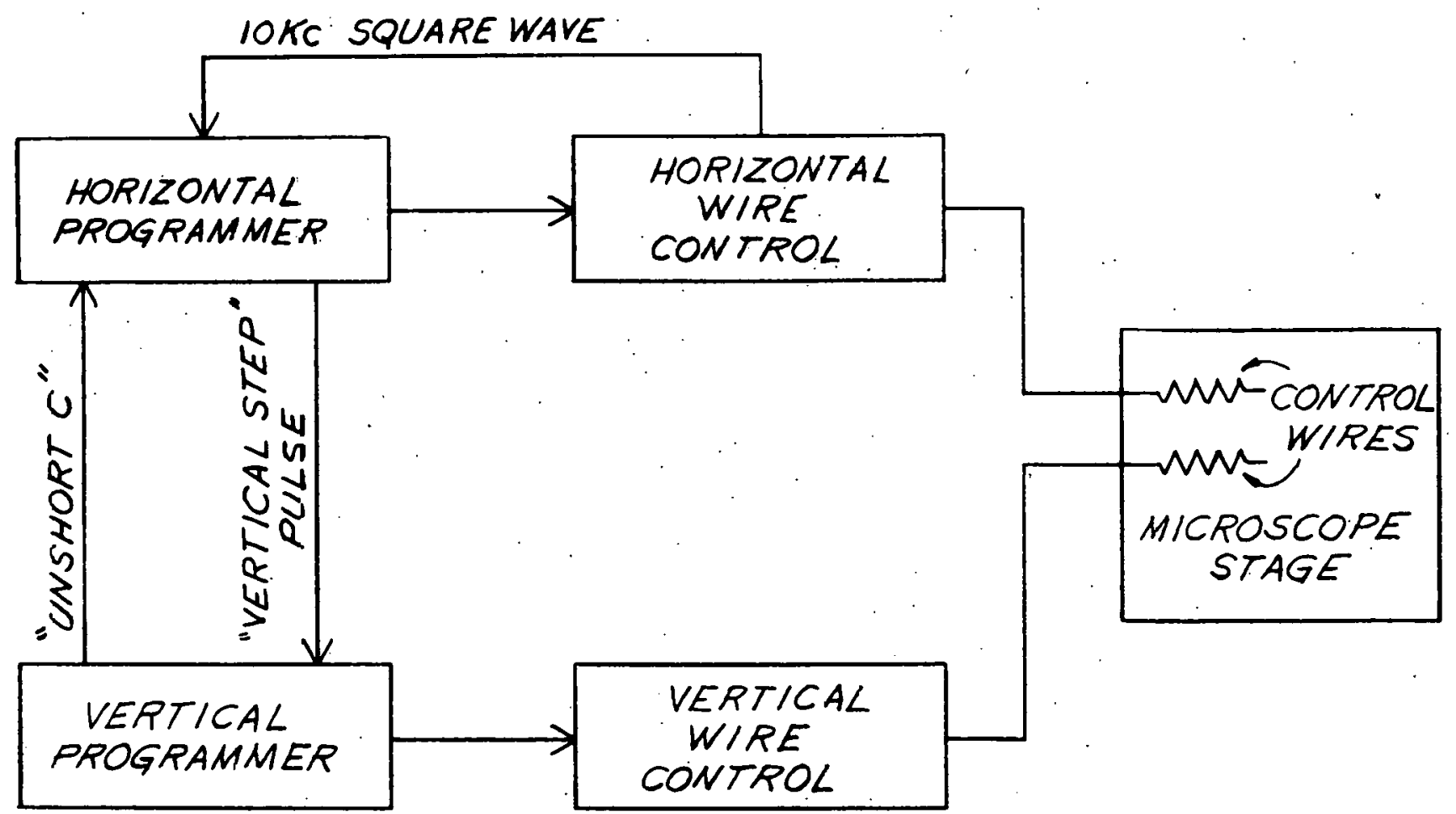

Fig. 4. Bloak diagram of electronic control system. 


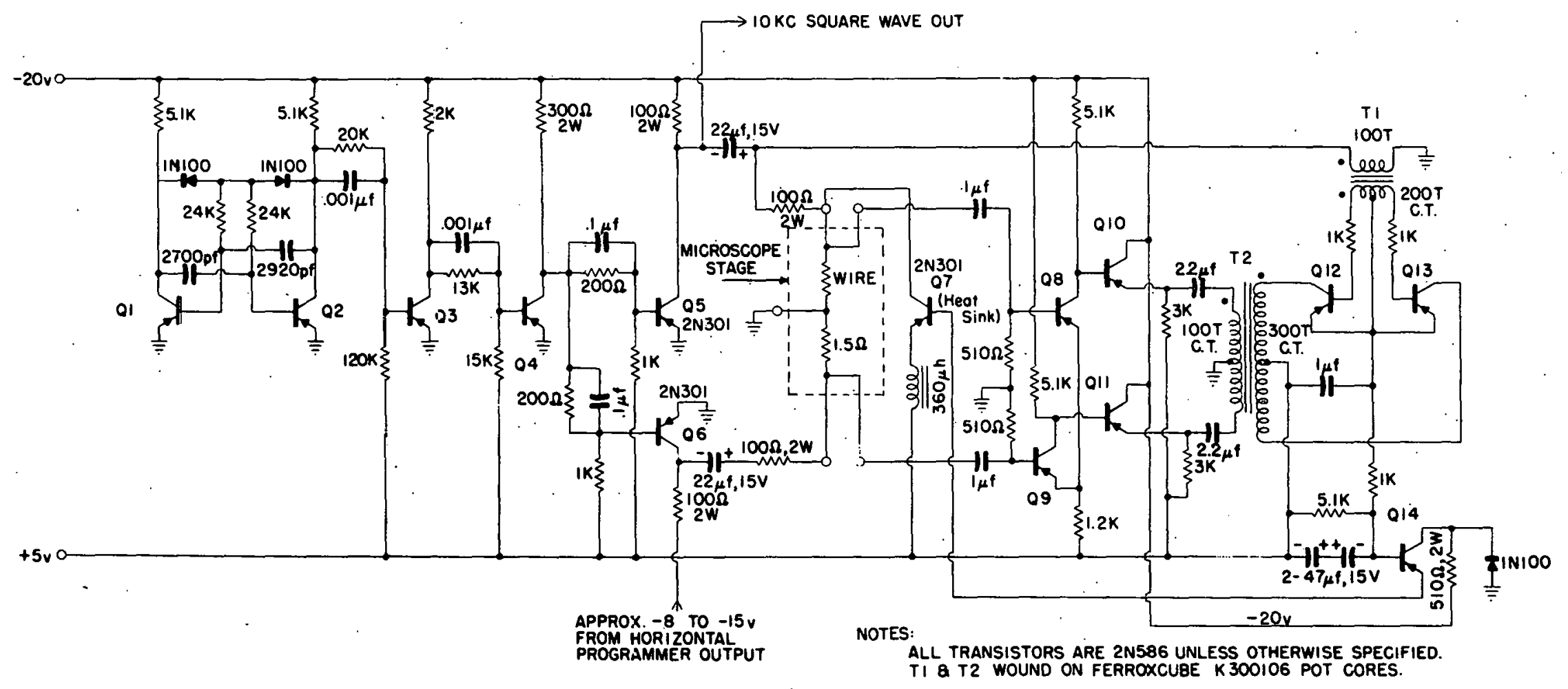

Fis. 5 Schematic of horizontal and vertical wire control circuit. 


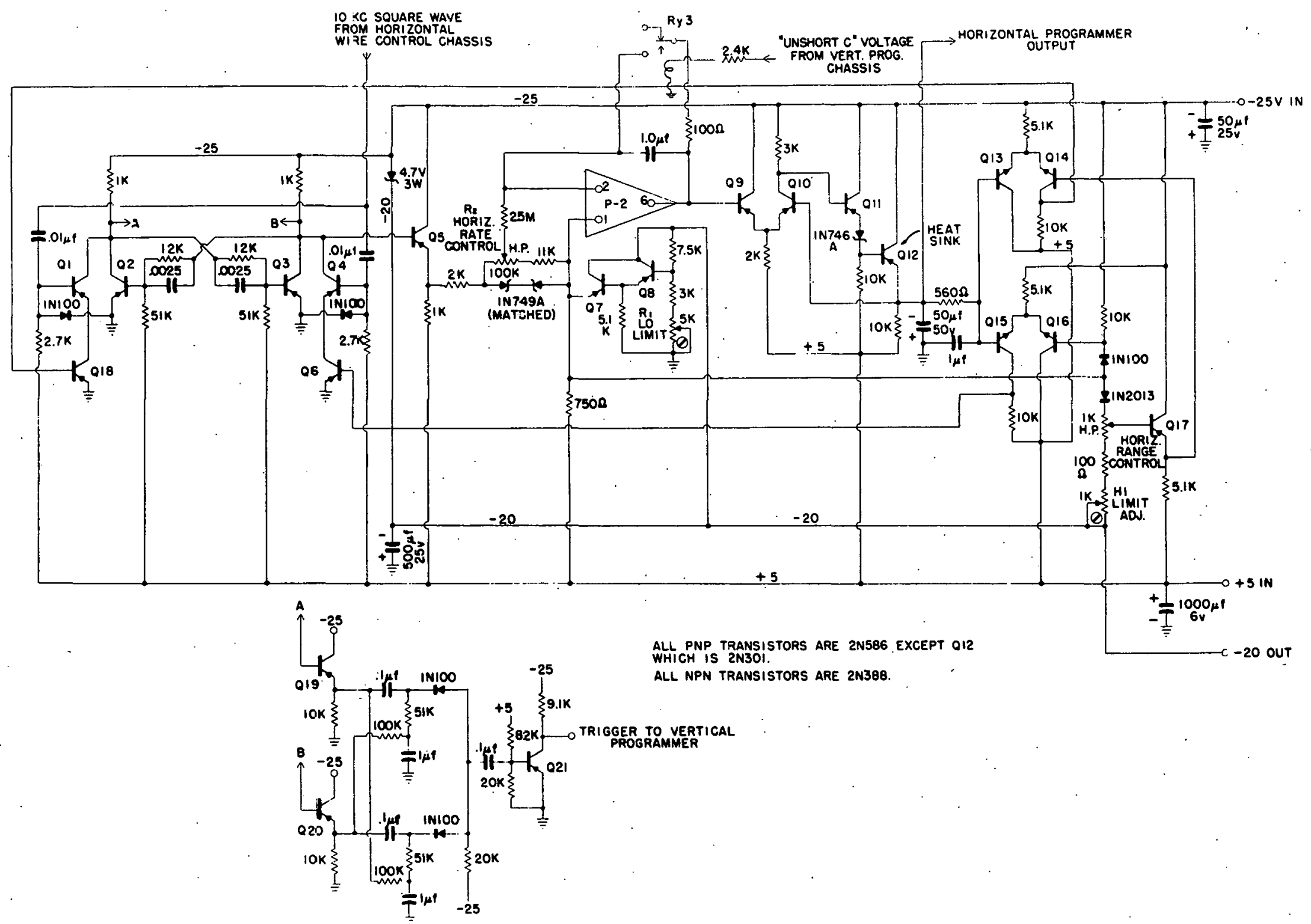

Fig. 6 Schematic of hirizontal programmer. 


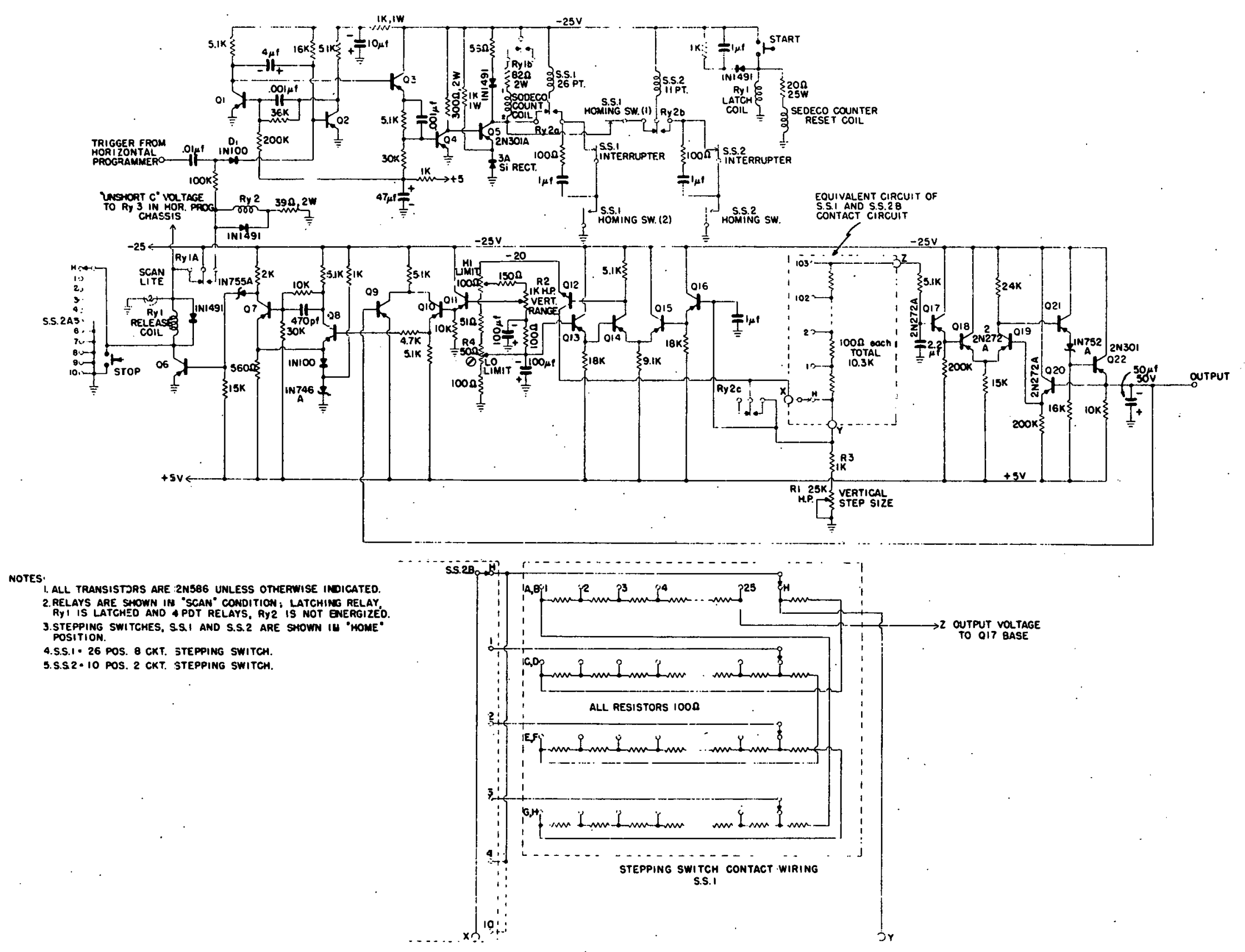

FiE. 7 Schematic of vertical programmer. 